\title{
THE CORRELATION BETWEEN PSYCHIATRIC DISORDERS AND COVID-19: A NARRATIVE REVIEW
}

\author{
Yanfei He ${ }^{1}, \operatorname{Ran} \mathbf{Y u}^{2} \&$ Junlin $\operatorname{Ren}^{3}$ \\ ${ }^{1}$ Health Management Center, the Sixth Medical Center, Chinese PLA General Hospital, Beijing, China \\ ${ }^{2}$ Department of Psychology, the Sixth Medical Center, Chinese PLA General Hospital, Beijing, China \\ ${ }^{3}$ Department of Infection Control, Sixth Medical Center, Chinese PLA General Hospital, Beijing, China
}

received: 1.3.2021;

revised: 29.3.2021;

accepted: 31.3.2021

\section{SUMMARY}

Since December 2019, the havoc caused by the severe acute respiratory syndrome coronavirus 2 (SARS-CoV-2) has increased exponentially in a short period of time. As the COVID-19 pandemic is raging around the world, scientists are trying to reveal its mysteriousness. Although COVID-19 is predominantly a respiratory disease, the most common symptoms are fever, dry cough, and fatigue, but extrapulmonary manifestations are increasingly recognized. Recent studies have shown that there is a strong genetic correlation between one or more psychiatric disorders and the occurrence of SARS-CoV-2 infection. Historical epidemiological perspectives and recent neurobiological evidence link infection and psychosis. What is the relationship between COVID-19 and psychiatric disorders? In this article, we will review the correlation between COVID-19 and psychoses, the possible reasons, and the possible pathophysiological mechanisms. The purpose of this review is to provide a reference for clinicians to make correct judgment and treatment when facing patients with COVID-19 and/or psychiatric disorders.

Key words: psychiatric disorder - COVID-19 - SARS-CoV-2 - psychiatry - neuropsychiatry

$$
* * * * *
$$

\section{INTRODUCTION}

COVID-19 pandemic is not only a great impact on almost all aspects of personal life and organization in the world (Cucinotta \& Vanelli 2020) but also an important source of psychological stress (TaubmanBen-Ari et al. 2020). The unpredictability and uncertainty of the COVID-19 epidemic, related blockades, physical distances, and other containment strategies, as well as the resulting economic collapse, may increase the risk of psychiatric disorders (Rajkumar 2020, Gupta et al. 2020). Recently, there have been more and more studies on the relationship between COVID-19 and psychoses (Nalleballe et al. 2020, Varatharaj et al. 2020). However, there is little information to date on a comprehensive review of the subject. In this review, we collect evidence of the associations between COVID-19 and psychoses, the possible reasons, and mechanisms. Our purpose is to remind clinicians that when facing patients suspected of COVID-19, they should consider that COVID-19 may be complicated with psychiatric disorders, to avoid misdiagnosis of COVID-19. At the same time, early diagnosis and treatment of psychoses in patients with COVID-19 can prevent further deterioration of late neuropsychiatric complications.

What is already known about this topic? - Many studies have shown that there are some associations between COVID-19 and psychiatric disorders.

What is added by this review? - COVID-19 may increase the incidence of psychiatric disorders and aggravate the severity of the original psychiatric disorders. And vice versa, patients with pre-existing psychiatric disorders are more susceptible to COVID-19 and can aggravate the severity of COVID-19. The etiology of the psychiatric consequences of the COVID-19 pandemic may be multifactorial. The possible pathophysiological mechanisms include direct viral infection, inflammatory reaction, autoimmunity, hypoxia, and hypercoagulable state.

What are the clinical implications for the future? Long-term follow-up and prospective studies are necessary to determine the real effect of COVID-19 on psychopathology.

\section{THE CORRELATION BETWEEN COVID-19 AND PSYCHIATRIC DISORDERS}

The arrival of a pandemic may become the main trigger for the beginning or aggravation of some harmful psychological characteristics, which may lead to behavioral/mental symptoms of clinical concern, which in turn will increase individual susceptibility to COVID-19 and aggravate the severity of COVID-19. Many studies have shown that COVID-19 is closely related to psychoses recently.

\section{COVID-19 may increase the incidence of psychiatric disorders and aggravate the severity of the original psychiatric disorders}

COVID-19 is a potentially fatal disease and a major source of stress for COVID-19 patients. During the pandemic, isolation, social distance, personal income instability caused by a sharp rise in unemployment, a shortage of social life resources, limited hospital resources, and barriers to access to health care 
caused by stigma, these are the main problems that emerged during the COVID-19 pandemic, all of them may lead to a negative impact on mental health (Richardson et al. 2020, Myers et al. 2020, Zhou et al. 2020), and people with mental disorders may be more vulnerable to these negative effects (Adhanom Ghebreyesus 2020, Li et al. 2020, Yao et al. 2020, Shinn \& Viron 2020). An observational study showed that the number of cases of schizophrenia increased by $25 \%$ in January 2020 compared with previous years, which was attributed to the psychosocial stress and physical distance measures related to the COVID-19 outbreaks (Hu et al. 2020). The review by Brown et al. postulated that there was a correlation between the high risk of psychiatric disorders and the psychosocial stress caused by the COVID-19 pandemic. The incidence of psychoses in confirmed COVID-19 patients is between $0.9 \%$ and $4 \%$ (Brown et al. 2020). Zheng noted a high prevalence of psychiatric disorders among survivors of the COVID-19 epidemic (Zheng 2020). A research showed that patients with obsessive-compulsive disorder (OCD) quarantined during the COVID-19 outbreak had higher obsessions and compulsions symptoms than before the outbreak (Davide et al. 2020).

National quarantine regulations during outbreaks make it more difficult and impractical to regularly go to outpatient clinics for evaluation and obtain prescription psychotropic substances, which can also increase the recurrence or deterioration of their pre-existing signs and symptoms (Mao et al. 2020). Also, COVID-19 infection per se and severe side effects of antiviral treatment may also aggravate some symptoms of psychiatric patients (Russell et al. 2020, Cortegiani et al. 2020, Sato et al. 2020). Individuals who suffer from mental health issues may be more especially vulnerable to the emotional response of the COVID-19 epidemic, resulting in worsening symptoms of pre-existing psychiatric disorders and/or an increased recurrence rate, because compared with the general population, they are highly sensitive to stress, such as alcohol substance use (Sun et al. 2020), eating disorders (Castellini et al. 2020). Symptoms of anxiety and depression, as well as highstress levels, were reported after the outbreak (Mazza et al. 2020).

\section{Patients with pre-existing psychiatric disorders are more susceptible to COVID-19 and can aggravate the severity of COVID-19}

Pre-existing psychiatric disorders may also change the individual susceptibility to COVID-19 (Holmes et al. 2020). When epidemics occur, people with psychiatric disorders are generally more vulnerable to infection (Batty et al. 2020). Emerging evidence suggests that there is a strong genetic link between at least one psychiatric diagnosis and the occurrence of infection, which provides additional support for this thought. Individuals with psychiatric disorders have a higher risk of developing COVID-19, especially the hospitalization and mortality associated with COVID-19. A variety of factors may contribute to a higher risk of SARS-CoV-2 infection and worse outcomes in people with psychiatric disorders (Nudel et al. 2019). First, psychiatric disorders may increase the risk of infections (Xiao 2020, Hamada \& Fan 2020). Possible explanations include cognitive impairment, lack of risk awareness, reduction in personal protection measures (such as hand washing, social distance, or isolation), and restrictions in psychiatric wards. Secondly, people with pre-existing mental disorders may face more barriers in accessing timely medical services after being infected with COVID-19 (Muruganandam et al. 2020), because of discrimination related to psychoses in the health care environment and their knowledge, skills, and access barriers to using telemedicine. Third, the coexistence of psychiatric disorders and COVID-19 will make the treatment more challenging and maybe less effective (Asmundson \& Taylor 2020). For example, people with known OCD may frequently self-monitor their temperature to check for fever, or they may try to swallow saliva several times to check for throat pain as a symptom of COVID-19. Hand-washing is an important preventive measure for COVID-19 transmission, which further aggravates the suffering of patients with known washer OCD. Finally, individuals with severe psychiatric disorders are more likely to suffer from co-existing diseases associated with a higher risk for severe COVID-19. Studies have shown that co-existing diseases (such as cancer, cardiovascular disease, obesity, type 2 diabetes) contributed to higher SARS-CoV-2 infection risk in patients with recent psychosis (Shinn \& Viron 2020). Patients with both COVID-19 and recently diagnosed psychiatric disorders have an increased risk of death (Wang et al. 2020).

\section{POSSIBLE REASONS}

The etiology of the psychiatric consequences of the current COVID-19 pandemic may be multifactorial, including brain infection, direct effects of cerebrovascular disease in a hypercoagulable state, response to pandemic-related stress (such as health intervention, social isolation, or stigma) (Wasserman et al. 2020), drug use (such as corticosteroids or antiviral drugs), and vertical transmission.

\section{Reactive response to pandemic-related stress}

The social distance and other public health interventions to curb the spread of COVID-19 may have an immediate and long-term impact on people with psychosis or people at risk of psychiatric disorder (Brown et al. 2020). During the pandemic, almost every country has developed public health strategies to curb the spread of COVID-19, including quarantine of people who may be exposed to the virus and requiring a physical distance from the general population (World Health Organization 2020). The incidence of depression and anxiety in 
people who implement social distance may be affected (Brooks et al. 2020). Despite telecommunications tools exist, distrust or unfamiliarity with technology may be additional pressure for people with mental disorders who use such tools. The COVID-19 pandemic has caused a considerable increase in unemployment rates, restricting individuals with psychiatric disorders from having a steady income, limiting their access to food, permanent housing, and medicine (Yancy 2020). Shortages of resources that patients rely on, such as transportation, groceries, and services from public buildings, further disrupt daily life. In addition, there are the impacts of reduced physical activity, dietary changes, and reduced sunlight exposure (Lippi et al. 2020). A study indicated that self-isolation was related to the increased C-reactive protein (CRP) level (Uchino et al. 2018). Busch et al. noted that individuals with greater stress were associated with higher inflammation levels (Busch et al. 2018). These results provide a basis for the conclusion of this study: self-isolation is a special form of inflammation. This can arise through psychosocial stressors irrespective of infection, for example, fear, isolation, bereavement, homelessness, broken relationships (divorce/separation), unemployment and financial difficulties, domestic violence, and deteriorating physical health. Given their susceptibility to social health determinants, all of these may have a particular effect on psychiatric patients (Odriozola-González et al. 2020, Wang et al. 2020, Anglin et al. 2020). Similarly, the direction of this effect is unclear, as pandemics have the potential to strengthen social inequalities, which in turn may lead to a risk of exceeding the consequences of the infection itself, further complicating the interaction between COVID-19 and psychiatric disorders.

\section{SARS-CoV-2 infection}

The effect of SARS-CoV-2 infection on the brain is associated with the excessive physiological and psychological stress of stimulating the hypothalamic-pituitaryadrenal axis, which further aggravates neuroinflammatory status. The duration and frequency of exposure to stressors impact neuroinflammation, which can be exacerbated by repeated or prolonged exposure to strong stressors (O'Callaghan \& Miller 2019). Longterm stress can enhance inflammatory response by releasing several pro-inflammatory cytokines. Neuroinflammation is an important aetiological factor for many neuropsychiatric and neurocognitive diseases, including neurodegenerative disorders (Bright et al. 2019), depression (Bakunina et al. 2015), psychosis (Marques et al. 2019), autism (Bjorklund et al. 2016), drug abuse (Kohno et al. 2019), and sleep disorders (Nadjar et al. 2017). A study on neuropsychiatric consequences of SARS conducted 30-50 months after infection showed an incidence of post-traumatic stress disorder (PTSD) was 40\%, depression was $36.4 \%$, OCD was $15.6 \%$, and with the same incidence of anxiety disorders (Troyer et al. 2020). Given this evidence, the sequelae of long-term post-SARS-CoV-2 psychiatric disorders are noteworthy. A recent study indicated the neuroinvasive nature of the virus and its possible route of transmission to the central nervous system by transmission electron microscopy of the brain tissue of a 74-year-old male patient with COVID-19 (Paniz-Mondolfi et al. 2020). It has clearly demonstrated that there are 80 to $110 \mathrm{~nm}$ viral particles in the vesicles of the frontal lobe and endothelial cells. Besides, the blebbing of viral particles in or out of the endothelial wall indicates the viral entry or exit across the brain microvascular endothelial cells into the nervous system.

\section{Corticosteroids and/or chloroquine and other antiviral drugs}

Corticosteroids usually induce psychiatric syndrome, including depression, mania, psychosis, and delirium, which is often referred to as 'steroid psychosis' and is considered to be a representative exogenous psychosis (Kershner \& Cheng 1989, Lewis \& Smith 1983). However, steroid psychosis is not a specific clinical entity but consists of heterogeneous syndromes with significantly different pathophysiological mechanisms. Psychiatric symptoms may also be secondary to side effects of drugs such as interferon and oseltamivir used to treat COVID-19 (Russell et al. 2020, Cortegiani et al. 2020). A pharmacovigilance study reported obviously neuropsychiatric side effects associated with chloroquine exposure, such as amnesia, hallucinations, depression, delirium, and loss of consciousness (Sato et al. 2020). The severity of COVID-19 symptoms and higher doses of corticosteroids were significantly related to an increased risk of experiencing psychiatric symptoms. Chloroquine may affect the dopaminergic system in the brain by changing dopamine levels, which in most cases is a high dopaminergic response. There is a substantial meta-analysis of evidence that proinflammatory cytokines are elevated in patients with primary psychosis. In fact, drugs targeting specific cytokines can cause psychosis (Pillinger et al. 2019, Goldsmith et al. 2016, Miller et al. 2011, Essali et al. 2019). High-dose steroid use has also been identified as a probable risk factor for psychosis, according to a case-control study in China (Lee et al. 2004).

\section{Encephalopathy and encephalitis}

Encephalopathy in COVID-19 may be the direct viral effect of neural invasion, an immune-mediated pathology induced by the virus, an indirect immunopathology caused by blood-brain barrier dysfunction, or a combination of all three. The virus enters the central nervous system by infecting endothelial cells of the blood-brain-barrier, epithelial cells of the blood-cerebrospinal fluid barrier in the choroid plexus, or by inflammatory cells ( $\mathrm{Li}$ et al. 2020, Toljan 2020). More importantly, the virus can reach the central nervous 
system by retrograde axonal transport. Retrograde axonal transport may be carried out through olfactory, respiratory, and enteric nervous system networks. After infecting the nasal cells, the virus can directly invade the brain, possibly through the olfactory bulbs, and rapidly spread to specific brain areas such as the thalamus and brainstem, triggering inflammation and demyelination (Bohmwald et al. 2018). SARS-CoV-2 has been detected in the cerebrospinal fluid of many patients with various psychiatric symptoms (Andriuta et al. 2020, Virhammar et al. 2020), it has provided undeniable evidence that SARS-CoV-2 can invade the nervous system. Two studies reported that $20 \%$ of patients had a complication of hypoxic encephalopathy following COVID-19, and patients who died were more likely to develop hypoxic encephalopathy than those who survived (Solomon et al. 2020, Rockx et al. 2020).

\section{Vertical transmission}

Several cross-sectional studies have shown that maternal stress, health anxiety, and social behavior interaction have changed significantly (Corbett et al. 2020, Mappa et al. 2020). The current COVID-19 pandemic may affect the fetal neurodevelopment of pregnant women during the pandemic by activating the immune system, directly due to maternal immune activation during infection, and indirectly due to inflammation exposed to maternal pressure during pregnancy. Inflammation is the common mediator of these two mechanisms. Isolation and limited movement may lead to a lack of social support from friends, relatives, and partners for pregnant women. Financial hardship, remote work, potentially violence from intimate partners, fewer pre-and post-natal appointments, and changes related to breastfeeding recommendations, which are a huge source of stress for perinatal women and their babies and can have short-and long-term harmful effects on pregnant women and babies (Burki 2020, The Lancet 2020). At the same time, studies have shown that vulnerable pregnant women are also highrisk groups with higher stress due to fear of infection or transmission of SARS-CoV-2 (Brooks et al. 2020, Knight et al. 2020). SARS-CoV-2 infection potentially triggers the maternal immune system to release a series of cytokines and chemokines (such as tumor necrosis factor-alpha, [TNF- $\alpha$ ] IL-6) and other immune changes that may be transmitted to the foetus. Besides, critically ill pregnant women caused by COVID-19 are at risk of placental hypoxia, damaging foetal oxygen supply, which may cause growth restriction and brain development stagnation. Studies have shown that maternal immune activation can lead to psychiatric disorders through multiple potential trajectories, thus making the offspring vulnerable to the environmental risks for psychosis (Khashan et al. 2008). A recent review summarises the mechanisms, including the effects associated with each trimester, the direct impacts of viruses reaching the foetus, and the effects of maternal and foetal immune activation (Yockey et al. 2020). IgG antibodies and cytokines may passively cross the placental barrier, while IgM usually does not due to its larger molecular structure, suggesting that antibodies may be produced by the infant to cope with intrauterine SARS-CoV-2 infection. One area of psychiatric research that may provide a precedent for the longitudinal effect of COVID-19 is the relationship between intrauterine events and neurodevelopment of offspring (Khashan et al. 2008, Brown 2012). Therefore, it will be important to closely monitor the outcomes of children whose mothers infected prior to, and during, pregnancy.

\section{CLINICAL MANIFESTATIONS OF PSYCHOSIS}

Psychosis is a state of serious disconnection between personal experience and reality or loss of self-boundaries. It is mainly manifested in the existence of hallucinations and delusions. Psychosis is a hallmark or defined symptom of schizophrenia spectrum and other mental disorders, and it is related to the characteristics of other mental and behavioral disorders. The systemic inflammation and neuroinflammatory changes are related to the massive increased brain proinflammatory molecules, glial reactivity, neurochemical landscape alternation, and pathological remodeling of neural networks. These organic changes are accompanied by environmental stress and promote the pathological disorder of neuropsychiatry.

Psychosis can be considered to be a set of symptoms, and there are a variety of manifestations in COVID-19 patients, ranging from insomnia and anxiety to psychiatric symptoms and aggressive behavior. This pattern of psychiatric symptoms is similar to those of patients infected with SARS in previous studies, ranging from mild mental problems (such as anger, anxiety, and depressive reactions) to severe psychotic problems such as hallucinations and mania (Lam et al. 2009, Cheng et al. 2004). A study shows that the most common mental symptoms of COVID-19 patients on admission are insomnia, followed by aggressive behavior, delusions, and severe anxiety (Xie et al. 2020). Besides, it is characterized by depression (Beurel et al. 2020), substance-related and addiction disorders (Alexander et al. 2020), eating disorders, schizophrenia (Brown et al. 2020), reactive psychosis, panic attacks, OCD, post-traumatic stress disorder, agoraphobia, and other psychiatric disorders.

\section{POSSIBLE PATHOPHYSIOLOGICAL MECHANISMS}

The postulated common pathophysiologic mechanisms by SARS-CoV-2 include direct viral infection, inflammatory response, autoimmunity, hypoxia, and hypercoagulable state. 


\section{SARS-CoV-2 Structure and ACE2 receptor}

COVID-19 pneumonia is caused by SARS-CoV-2 (He et al. 2020), as the seventh known coronavirus that can infect humans (humans coronavirus, H-CoV). The first six coronaviruses that can cause severe diseases in humans include Middle East Respiratory Syndrome (MERS)-CoV, HCoV-HKU1, HCoV-OC43, HCoVNL63, SARS-CoV, and HCoV-229E (Corman 2019). The SARS-CoV-2 virion is approximate with a size of 70-100 nm (Naqvi et al. 2020). Its genome encodes four main structural proteins: Spike (S), envelope (E), membrane (M), and nucleocapsid $(\mathrm{N})$. Recent studies have shown that some coronaviruses do not require all four proteins to become infectious virions (Schoeman \& Fielding 2019).

ACE2 receptors are recognized as the SARS-CoV-2 receptor so far, which exist in a variety of human tissues, including type I and II alveolar epithelial cells, vascular endothelial cells, heart, liver, gastrointestinal tract (Hamming et al. 2004), as well as kidneys and testicles (Fan et al. 2020). It also presents at the cellular level of the central nervous system, such as the spinal cord, cortex, hippocampus, and cerebellum (Xia \& Lazartigues 2008, Buzhdygan et al. 2020). S and E proteins play a critical role in aiding SARS-CoV-2 S proteins to interact with ACE2 receptors expressed in capillary endothelium (Baig et al. 2020).

\section{Direct infection}

The first step of SARS-CoV-2 infection is the recognition of host cell surface receptors. This step is mediated by the viral $\mathrm{S}$ protein, which recognizes the human receptor angiotensin-converting enzyme 2 (ACE2). The protein has two functional domains: the S1 domain contains the receptor-binding domain (RBD), which binds to ACE2, while the S2 domain mediates the fusion of the virus and the host cellular membrane (Walls et al. 2020). Therefore, the organ distribution of the ACE2 receptor is an important determinant of the virus infectivity and tropism. The second determinant step of the SARS-CoV-2 infection process is the activation of the $\mathrm{S}$ protein. This protein processing allows the complete activity of the S2 domain and the fusion of the viral and cellular membrane. The SARSCoV-2 enters the cell following binding to the plasmalemmal ACE2 enzyme and then endocytosis (Liu et al. 2020, Lu et al. 2020). Literature suggests the enriched presence of angiotensin-converting enzyme 2 (ACE2) in the central nervous system (CNS) endothelial cells.91 Possibly the virus infects these endothelial cells in the CNS by crossing the blood-brain barrier (Solomon et al. 2020, Deffner et al. 2020). The neuronal retrograde mode is also suggested in a few studies, in which the virus initially invades the peripheral nervous system followed by infecting the CNS (Wang et al. 2020, Paniz-Mondolfi et al. 2020), neurotropic viruses may invade neurons through virion retrograde or/and anterograde interactions with neuronal cytoskeleton proteins. After neuronal infection, the virions may be released and transneuronal spread to adjacent or presynaptic neurons (Butowt \& Bilinska 2020).

\section{Inflammatory reaction}

A growing body of evidence suggests that psychiatric disorders of COVID-19 patients may be associated with the level of inflammatory markers. It is well known that COVID-19 can cause increased levels of serum inflammatory markers by activating an immune response (Azkur et al. 2020). Meta-analysis data confirmed that there was a significant increase in pro-inflammatory molecules in patients with post-traumatic stress disorder, including IL-6, TNF- $\alpha$, and IL-1 $\beta$ (Lindqvist et al. 2017, Bersani et al. 2016). However, the significance of the acute inflammatory state in the occurrence and development of neuropsychiatric sequela is unclear, which stems not only from the severity of systemic inflammation and the invasion of the brain by the virus but also from the severity of stress caused by unexpected pandemics (Northoff 2002). The alterations of GABA-ergic and dopaminergic regulation in the cortico-basal ganglia-thalamocortical circuit are related to its pathogenesis (Rossi et al. 2011). Exposure to pro-inflammatory cytokines is associated with altered GABA-ergic transmission in the basal ganglia. Other pro-inflammatory mediators such as interferon-alpha are considered to be associated with a hypo-dopaminergic state in the basal ganglia, which is considered to be a potential inducing factor to psychiatric disorders. Also, the hematological high inflammatory state of COVID-19 patients increases the blood-brain barrier permeability, destroys brain cells, and becomes a promoting factor for the occurrence of psychoses. A recent report on a case of psychosis patient with COVID-19 further implicates that the hyper-inflammatory state is related to the pathogenesis of these neuropsychiatric complications of severe COVID-19 (Kajani et al. 2020). Besides, inflammation is an example of a common biological factor that contributes to various psychiatric disorders and COVID-19 pathology. It is reported that inflammation plays a role in the pathogenesis of schizophrenia (Müller 2018), depression (Beurel et al. 2020), and bipolar disorder (Benedetti et al. 2020), as well as in the systemic manifestations of SARS-CoV-2 infection (Steardo et al. 2020).

\section{Autoimmunity}

When an individual with immune function is infected with SARS-CoV-2, he may experience excessive systemic immune responses and even die of acute respiratory distress syndrome and septic shock ( $\mathrm{Li}$ et al. 2020). This mechanism is called immunopathogenicity, in which the virus stimulates immunity and causes the immune system to attack itself (Guo \& Thomas 2017). The excessive activation of the immune system caused by SARS-CoV-2 infection leads to 
massive accumulation and exudation of inflammatory substances, which is known as 'cytokine storm'. Cytokine storm may cause a sharp increase of monocytes, interferons, interleukins, macrophage inflammatory proteins, and tumor necrosis factors, leading to hyper-inflammation. This systemic inflammation causes severe encephalopathy, which can lead to symptoms of psychiatric disorders. A high level of IL-1 $\beta$ has been observed in COVID-19 patients, and other immune mediators including IL-2, IL-6, IL-7, IL-10 (Wan et al. 2020), and granulocyte colony-stimulating factor (G-SCF), which have strong pro-inflammatory effects and can cause neuroinflammation and brain dysfunction (Poyiadji et al. 2020).

\section{Hypoxia}

COVID-19 patients often have hypoxemia caused by respiratory dysfunction (Wang et al. 2020, Cascella et al. 2020), among which severe patients are characterized by acute respiratory distress syndrome (Murthy et al. 2020). When the bodily oxygen content changes, the brain is the first organ to be affected (Casas et al. 2017), resulting in a series of pathophysiological changes. Hypoxia can dilate intracranial blood vessels, increase intracranial blood flow, cerebral capillary pressure, tissue fluid production, brain free radicals, membrane lipid peroxidation, and endogenous inhibitors, which may affect cell energy metabolism (Mikhail Kellawan et al. 2017, Numagami et al. 1997). The increased lactic acid and oxygen free radicals and lipid peroxides produced by anaerobic glycolysis in the brain, and the weakened antioxidant system lead to blood-brain barrier dysfunction (Rosenkrantz et al. 1996). Metabolic acidosis due to hypoxia increases cerebral vasospasm and permeability, resulting in interstitial brain edema and intracranial hypertension (Boedtkjer 2018), which can lead to a series of injuries, culminating in neuronal and astroglial necrosis, apoptosis, and neurocognitive deficits (Mallet \& Ryou 2017).

\section{Hypercoagulable state}

Clinical studies showed that the elevated D-dimer (Tang et al. 2020), the prolonged prothrombin time with unchanged activated partial thromboplastin time (APTT), and increased fibrinogen are commonly observed in patients with COVID-19, and the blood was in a state of hypercoagulability. One possible explanation for the increased blood coagulability is that a decreased blood oxygen activates increased the levels of inflammatory cytokines, catecholamine, plasma tissue factors (Kammerer et al. 2020), and platelet aggregation, which change capillary blood flow because of increased sympathetic activity and broader micro-endothelial damage, leading to increased blood coagulation (Toraldo et al. 2015), and capillary microthrombosis (Suresh et al. 2019), which may cause ischemic damages of small vessels in the brain, leading to psychoses.

\section{PROSPECTS FOR THE FUTURE}

In the long term, as symptoms and potential effects of COVID-19 continue to manifest, patients may be left with long-term neurological and/or psychiatric disorders on a global scale (Sinanovic et al. 2020, Roy et al. 2020). The role of intrauterine infection in the neurodevelopment of offspring born during this pandemic may not become apparent for many years for both infected and non-infected mothers. Notably, knowledge of the long-term psychiatric effects of COVID-19 on surviving patients is almost absent. The psychiatric sequelae of COVID-19 represent a serious clinical challenge, which has to be considered in complex screening, treatment, and follow-up in the future. It is necessary to conduct a long-term follow-up and a prospective study on the direct biological impact of COVID-19 on psychoses, in order to more accurately describe the incidence of psychoses related to SARSCoV-2 during and after infection, as well as the real effect and potential pathogenesis of SARS-CoV-2 on psychopathology.

\section{LIMITATIONS}

This review has several limitations. First, most of the current papers on clinical features were designed retrospectively, which may introduce potential biases. Second, the symptoms associated with psychoses came from limited sample size observations and case studies, some of which were not classical psychiatric manifestations, and the psychiatric participation may contribute partially to these symptoms, and an overall analysis may exaggerate its role in COVID-19. Third, many involved studies have not yet been published, which may affect the results. The potential mechanism underlying psychiatric disorders in COVID-19 will be updated along with new evidence.

\section{CONCLUSIONS}

The COVID-19 pandemic poses a long-lasting challenge, which not only impacts the cardiopulmonary system but links systemic infection to psychiatric disorders. When doctors find the symptoms of psychoses in COVID-19 patients, they should always consider differential diagnosis to avoid delay or misdiagnosis. Long-term follow-up is necessary to determine the future incidence of psychiatric disorders after SARS-COV-2 infection, and prospective studies should be conducted to find out the real impact of COVID-19 on psychopathology, and finally overcome the epidemic of COVID-19.

Acknowledgments: None.

Conflict of interest: None to declare. 


\section{Contribution of individual authors:}

Yanfei He designed the study, reviewed literature, and drafted the manuscript.

Ran $\mathrm{Yu}$ advised on the review and reviewed the final manuscript.

Junlin Ren retrieved and summarized the literature.

All authors read and approved the final manuscript.

\section{References}

1. Adhanom Ghebreyesus T: Addressing mental health needs: an integral part of COVID-19 response. World Psychiatry 2020; 19:129-30

2. Alexander GC, Stoller KB, Haffajee RL, Saloner B: An epidemic in the midst of a pandemic: opioid use disorder and COVID-19. 2020

3. Andriuta D, Roger P-A, Thibault W, et al.: COVID-19 encephalopathy: detection of antibodies against SARSCoV-2 in CSF. Journal of Neurology 2020; 267

4. Anglin DM, Galea S, Bachman P: Going upstream to advance psychosis prevention and improve public health. JAMA Psychiatry 2020; 77:665-666

5. Asmundson GJG, Taylor S: How health anxiety influences responses to viral outbreaks like COVID-19: what all decision-makers, health authorities, and health care professionals need to know. J Anxiety Disord 2020; 71:102211

6. Azkur AK, Akdis M, Azkur D. et al.: Immune response to SARS-CoV-2 and mechanisms of immunopathological changes in COVID-19. Allergy 2020; 75:1564-1581

7. Baig AM, Khaleeq A, Ali U, Syeda H: Evidence of the COVID-19 virus targeting the CNS: Tissue distribution, host-virus interaction, and proposed neurotropic mechanisms. ACS Chem Neurosci 2020; 11:995-998

8. Bakunina N, Pariante CM, Zunszain PA: Immune mechanisms linked to depression via oxidative stress and neuroprogression. Immunology 2015; 144:365-373

9. Batty GD, Deary IJ, Luciano M, et al.: Psychosocial factors and hospitalisations for COVID-19: Prospective cohort study based on a community sample. Brain Behav Immun 2020; 89:569-578

10. Benedetti F, Aggio V, Pratesi ML, et al.: Neuroinflammation in bipolar depression. Front Psychiatry 2020; 11:71

11. Bersani FS, Wolkowitz OM, Lindqvist D, et al.: Global arginine bioavailability, a marker of nitric oxide synthetic capacity, is decreased in PTSD and correlated with symptom severity and markers of inflammation. Brain Behav Immun 2016; 52:153-160

12. Beurel E, Toups M, Nemeroff CB: The bidirectional relationship of depression and inflammation: double trouble. Neuron 2020; 107:234-56

13. Bjorklund $G$, Saad K, Chirumbolo $S$, et al.: Immune dysfunction and neuroinflammation in autism spectrum disorder. Acta Neurobiol Exp (Wars) 2016; 76:257-268

14. Boedtkjer E: Acid-base regulation and sensing: accelerators and brakes in metabolic regulation of cerebrovascular tone. J Cereb Blood Flow Metab 2018; 38:588602

15. Bohmwald K, Gálvez NMS, Ríos M, Kalergis AM: Neurologic alterations due to respiratory virus infections. Front Cell Neurosci 2018; 12:386
16. Bright F, Werry EL, Dobson-Stone C, et al.: Neuroinflammation in frontotemporal dementia. Nat Rev Neurol 2019; 15:540-555

17. Brooks SK, Webster RK, Smith LE, et al.: The psychological impact of quarantine and how to reduce it: rapid review of the evidence. Lancet 2020; 395:912-920

18. Brown AS: Epidemiologic studies of exposure to prenatal infection and risk of schizophrenia and autism. Dev Neurobiol 2012; 72:1272-6

19. Brown E, Gray R, Monaco SL, Thompson A, et al.: The potential impact of COVID-19 on psychosis: a rapid review of contemporary epidemic and pandemic research. Schizoph Res 2020; 222:79-87

20. Burki T: The indirect impact of COVID-19 on women. Lancet Infect Dis 2020; 20:904-5

21. Busch EL, Whitsel EA, Kroenke CH, Yang YC: Social relationships, inflammation markers, and breast cancer incidence in the Women's Health Initiative. Breast 2018; 39:63-69

22. Butowt R, Bilinska K: SARS-CoV-2: Olfaction, Brain Infection, and the Urgent Need for Clinical Samples Allowing Earlier Virus Detection. ACS Chem Neurosci 2020; 11:1200-3

23. BuzhdyganTP, DeOre BJ, Baldwin-Leclair A, et al:: The SARS-CoV-2 spike protein alters barrier function in $2 D$ static and $3 D$ microfluidic in-vitro models of the human blood-brain barrier. RevMed Liege 2020; 75(S1):130-132

24. Casas AI, Geuss E, Kleikers PWM, et al.: NOX4-dependent neuronal autotoxicity and BBB breakdown explain the superior sensitivity of the brain to ischemic damage. Proc Natl Acad Sci USA 2017; 114:12315-12320

25. Cascella M, Rajnik M, Cuomo A,Dulebohn SC, Di Napoli $R$ : Features, evaluation and treatment coronavirus (COVID-19) (StatPearls.Internet]). StatPearls Publishing; Oct 4, 2020. Available from: https://www.ncbi.nlm.nih.gov/books/NBK554776/

26. Castellini G, Cassioli E, Rossi E, et al.: The impact of COVID-19 epidemic on eating disorders: a longitudinal observation of pre versus post psychopathological features in a sample of patients with eating disorders and a group of healthy controls. Int J Eating Disord 2020; 53:18551862

27. Cheng SK, Tsang JS, Ku KH, Wong CW, Ng YK: Psychiatric complications in patients with severe acute respiratory syndrome (SARS) during the acute treatment phase: a series of 10 cases. Br J Psychiatry 2004; 184:359-360

28. Corbett GA, Milne SJ, Hehir MP, Lindow SW, O'connell MP: Health anxiety and behavioural changes of pregnant women during the COVID-19 pandemic. Eur J Obstet Gynecol Reprod Biol 2020; 249:96-7

29. Corman VM, Lienau J, Witzenrath M: Coronaviren als Ursache respiratorischer Infektionen. In Der Internist (Berl) 2019; 60:1136-45

30. Cortegiani A, Ingoglia G, Ippolito M, Giarratano A, Einav S: A systematic review on the efficacy and safety of chloroquine for the treatment of COVID-19. J Crit Care 2020; 57:279-283

31. Cucinotta D, Vanelli M. WHO declares COVID-19 a pandemic. Acta Biomed 2020; 91:157-60

32. Davide P, Andrea P, Martina O, Andrea E, Davide D, Mario A: The impact of the COVID-19 pandemic on patients with OCD: effects of contamination symptoms and remission state before the quarantine in a preliminary naturalistic study. Psychiatry Res 2020; 9:113213 
33. Deffner F, Scharr M, Klingenstein S, et al.: Histological Evidence for the Enteric Nervous System and the Choroid Plexus as Alternative Routes of Neuroinvasion by SARSCoV2. Front Neuroanat 2020; 14:596439

34. Essali N, Goldsmith DR, Carbone L, Miller BJ: Psychosis as an adverse effect of monoclonal antibody immunotherapy. Brain Behav Immun 2019; 81:646-9

35. Fan C, Li K, Ding Y, Lu WL, Wang J: ACE2 expression in kidney and testis may cause kidney and testis damage after 2019-nCoV infection, medRxiv. https://doi.org/10.1101/2020.02.12.20022418

36. Goldsmith DR, Rapaport MH, Miller BJ: A meta-analysis of blood cytokine network alterations in psychiatric patients: comparisons between schizophrenia, bipolar disorder and depression. Mol Psychiatry 2016; 21:1696-1709

37. Guo XJ, Thomas PG: New fronts emerge in the influenza cytokine storm. Semin Immunopathol 2017; 39:541-550

38. Gupta A, Madhavan MV, Sehgal K, et al.: Extrapulmonary manifestations of COVID-19. Nature Medicine 2020; 26:1017-1032

39. Hamada K, Fan X: The impact of COVID-19 on individuals living with serious mental illness. Schizophrenia research 2020; 222:3-5

40. Hamming I, Timens $W$, Bulthuis $M$, Navis $G$, van Goor H: Tissue distribution of ACE2 protein, the functional receptor for SARS coronavirus. A first step in understanding SARS pathogenesis. J Pathol 2004; 203:631-637

41. He F, Deng Y, Li W: Coronavirus disease 2019 (COVID19): What we know? J Med Virol 2020; 92:719-725

42. Holmes EA, O'Connor RC, Perry VH, et al.: Multidisciplinary research priorities for the COVID-19 pandemic: A call for action for mental health science. Lancet Psychiatry 2020; 7:547-560

43. Hu W, Su L, Qiao J, et al.: COVID-19 outbreak increased risk of schizophrenia in aged adults. Chinaxiv.org (preprint). Available from: http://chinaxiv.org/abs/202003.00003

44. Kajani R, Apramian A, Vega A, Ubhayakar N, Xu P, Liu A: Neuroleptic malignant syndrome in a COVID-19 patient. Brain, Behavior Immun 2020; 88:28-29

45. Kammerer T, Faihs V, Hulde N, et al.: Hypoxic-inflammatory responses under acute hypoxia: in vitro experiments and prospective observational expedition trial. Int $\mathrm{J} \mathrm{Mol}$ Sci 2020; 21:1034. doi:10.3390/ijms21031034

46. Kershner P, Cheng RW: Psychiatric side effects of steroid therapy. Psychosomatics 1989; 30:135-139

47. Khashan AS, Abel KM, McNamee R, et al.: Higher Risk of Offspring Schizophrenia Following Antenatal Maternal Exposure to Severe Adverse Life Events. Arch Gen Psychiatry 2008; 65:146-52

48. Knight M, Bunch K, Vousden N, et al.: Characteristics and outcomes of pregnant women admitted to hospital with confirmed SARS-CoV-2 infection in UK: National population based cohort study. BMJ 2020; 369:m2107

49. Kohno M, Link J, Dennis LE, et al.: Neuroinflammation in addiction: a review of neuroimaging studies and potential immunotherapies. Pharm Biochem Behav 2019; 179:34-42

50. Lam MH, Wing YK, Yu MW, et al.: Mental morbidities and chronic fatigue in severe acute respiratory syndrome survivors: long-term follow-up. Arch Intern Med 2009; 169:2142-2147

51. Lee DTS, Wing YK, Leung HCM, et al.: Factors associated with psychosis among patients with severe acute respiratory syndrome: a case-control study. Clin Infect Dis 2004; 39:1247-1249

52. Lewis DA, Smith RE: Steroid-induced psychiatric syndromes. J Affect Dis 1983; 5:319-332

53. Li G, Fan Y, Lai Y, et al.: Coronavirus infections and immune responses. $J$ Med Virol 2020; 92:424-432

54. Li J, Yang Z, Qiu H, et al.: Anxiety and depression among general population in China at the peak of the COVID-19 pandemic. World Psychiatry 2020; 19:249-50

55. Lindqvist D, Dhabhar FS, Mellon SH, et al.: Increased pro-inflammatory milieu in combat related PTSD-A new cohort replication study. Brain Behav Immun 2017; 59:260-264

56. Lippi G, Henry BM, Sanchis-Gomar F: Physical inactivity and cardiovascular disease at the time of coronavirus disease 2019 (COVID-19). Eur J Prev Cardiol 2020; 27:906-908

57. Lu R, Zhao X, Li J, et al.: Genomic characterisation and epidemiology of 2019 novel coronavirus: implications for virus origins and receptor binding. Lancet 2020; 395:565-574

58. Liu Z, Xiao X, Wei X, et al.: Composition and divergence of coronavirus spike proteins and host ACE2 receptors predict potential intermediate hosts of SARS-CoV-2. J Med Virol 2020; 92:595-601

59. $L i$ YC, Bai WZ, Hashikawa T: The neuroinvasive potential of SARS-CoV2 may play a role in the respiratory failure of COVID-19 patients. J Med Virol 2020; 92:552-555

60. Mallet RT, Ryou MG: Erythropoietin: endogenous protection of ischemic brain. Vitam Horm 2017; 105:197-232

61. Mao L, Wang M, Chen S, et al.: Neurological manifestations of hospitalized patients with COVID-19 in Wuhan, China: a retrospective case series study. SSRN J 2020; e201127

62. Mappa I, Distefano FA, Rizzo G: Effects of coronavirus 19 pandemic on maternal anxiety during pregnancy: a prospectic observational study. J Perinat Med 2020; 48:545-50

63. Marques TR, Ashok AH, Pillinger T, et al.: Neuroinflammation in schizophrenia: meta-analysis of in vivo microglial imaging studies. Psychol Med 2019; 49:21862196

64. Mazza C, Ricci E, Biondi S, et al.: A nationwide survey of psychological distress among Italian people during the COVID-19 pandemic: Immediate psychological responses and associated factors.Int $J$ Environ Res Public Health 2020; 17

65. Mikhail Kellawan J, Harrell JW, Roldan-Alzate A, Wieben $O$, Schrage WG: Regional hypoxic cerebral vasodilation facilitated by diameter changes primarily in anterior versus posterior circulation. J Cereb Blood Flow Metab 2017; 37:2025-2034

66. Miller BJ, Buckley P, Seabolt W, Mellor A, Kirkpatrick B: Meta-analysis of cytokine alterations in schizophrenia: clinical status and antipsychotic effects. Biol Psychiatry 2011; 70:663-71

67. Müller N: Inflammation in schizophrenia: pathogenetic aspects and therapeutic considerations. Schizophr Bull 2018; 44:973-82

68. Murthy S, Gomersall CD, Fowler RA: Care for critically ill patients with COVID-19. JAMA 2020; 323:1499

69. Muruganandam $P$, Neelamegam S, Menon $V$, et al.: COVID-19 and Severe Mental Illness: Impact on patients 
and its relation with their awareness about COVID-19. Psychiatry Research 2020; 291:113265

70. Myers LC, Parodi SM, Escobar GJ, et al.: Characteristics of hospitalized adults with COVID-19 in an integrated health care system in California. JAMA 2020; 323:2195-8

71. Nadjar A, Wigren HM, Tremblay ME: Roles of Microglial Phagocytosis and Inflammatory Mediators in the Pathophysiology of Sleep Disorders. Front Cell Neurosci 2017; 11:250

72. Nalleballe K, Reddy Onteddu S, Sharma R, et al.: Spectrum of neuropsychiatric manifestations in COVID-19. Brain Behav Immun 2020; 88:71-74

73. Naqvi AAT, Fatima K, Mohammad T, et al.: Insights into SARS-CoV-2 genome, structure, evolution, pathogenesis and therapies: Structural genomics approach. BBA Molecular Basis of Disease. [published online, June 13, 2020]. $\quad$ https://doi.org/10.1016/j.bbadis.2020.165878. Accessed 21 January 2021

74. Northoff G: What catatonia can tell us about "top-down modulation": a neuropsychiatric hypothesis. Behav Brain Sci 2002; 25:555-577

75. Nudel R, Wang Y, Appadurai V, et al.: A large-scale genomic investigation of susceptibility to infection and its association with mental disorders in the Danish population. Transl Psychiatry 2019; 9:283

76. Numagami Y, Zubrow AB, Mishra OP, Delivoria-Papadopoulos M: Lipid free radical generation and brain cell membrane alteration following nitric oxide synthase inhibition during cerebral hypoxia in the newborn piglet. $J$ Neurochem 1997; 69:1542-1547

77. O'Callaghan JP, Miller DB: Neuroinflammation disorders exacerbated by environmental stressors. Metabolism 2019; 100S: 153951

78. Odriozola-González P, Planchuelo-Gómez Á, Irurtia MJ, de Luis-Garcia R: Psychological effects of the COVID-19 outbreak and lockdown among students and workers of a Spanish university. Psychiatry Res 2020; 290:113108

79. Paniz-Mondolfi A, Bryce C, Grimes Z, et al.: Central nervous system involvement by severe acute respiratory syndrome coronavirus-2 (SARS-CoV-2). J Med Virol 2020; 92:699-702

80. Pillinger $T$, Osimo EF, Brugger $S$, Mondelli $V$, McCutcheon RA, Howes OD: A Metaanalysis of Immune Parameters, Variability, and Assessment of Modal Distribution in Psychosis and Test of the Immune Subgroup Hypothesis. Schizophr Bull 2019; 45:11201133

81. Poyiadji N, Shahin G, Noujaim D, et al.: COVID-19 associated acute hemorrhagic necrotizing encephalopathy: Imaging features. Radiology 2020; 296:E119-E120

82. Rajkumar RP: COVID-19 and mental health: A review of the existing literature. Asian Journal of Psychiatry 2020; 52:102066

83. Richardson $S$, Hirsch JS, Narasimhan $M$, et al.: Presenting characteristics, comorbidities, and outcomes among 5700 patients hospitalized with COVID-19 in the New York City area. JAMA 2020; 323:2052-9

84. Rockx B, Kuiken T, Herfst $S$, et al.: Comparative pathogenesis of COVID-19, MERS, and SARS in a nonhuman primate model. Science 2020; 368:1012-5

85. Rosenkrantz TS, Kubin J, Mishra OP, Smith D, DelivoriaPapadopoulos M: Brain cell membrane $\mathrm{Na}+, \mathrm{K}(+)$ ATPase activity following severe hypoxic injury in the newborn piglet. Brain Res 1996; 730:52-57
86. Rossi $S$, Muzio L, De Chiara V, et al.: Impaired striatal GABA transmission in experimental autoimmune encephalomyelitis. Brain, Behavior, Immunity 2011; 25:947-956

87. Roy D, Ghosh R, Dubey $S$, et al.: Neurological and Neuropsychiatric Impacts of COVID-19 Pandemic. Can J Neurol Sci 2020; 1-16

88. Russell CD, Millar JE, Baillie JK, J.E, Baillie, J.K: Clinical evidence does not support corticosteroid treatment for 2019-nCoV lung injury. Lancet 2020; 395:473-475

89. Sato K, Mano T, Iwata A, Toda T: Neuropsychiatric adverse events of chloroquine: a real-world pharmacovigilance study using the FDA Adverse Event Reporting System (FAERS) database. Biosci Trends 2020; 14:139143

90. Schoeman D, Fielding BC: Coronavirus envelope protein: current knowledge. Virol J 2019; 16:69

91. Shinn AK, Viron M: Perspectives on the COVID-19 pandemic and individuals with serious mental illness. J Clin Psychiatry 2020; 81:20com13412

92. Sinanovic O, Muftic M, Sinanovic S: COVID-19 Pandemia: Neuropsychiatric Comorbidity and Consequences. Psychiatr Danub 2020; 32:236-44

93. Solomon IH, Normandin E, Bhattacharyya $S$, et al.: Neuropathological Features of Covid-19. N Engl J Med 2020; 383:989-92

94. Steardo L Jr, Steardo L, Verkhratsky A: Psychiatric face of COVID-19. Transl Psychiatry 2020; 10:261

95. Sun Y, Li Y, Bao Y, et al.: Brief report: increased addictive internet and substance use behavior during the COVID-19 pandemic in China. Am J Addict 2020; 29:268-270

96. Suresh S, Rajvanshi PK, Noguchi CT: The Many Facets of Erythropoietin Physiologic and Metabolic Response. Front Physiol 2019; 10:1534

97. Tang $N$, Bai $H$, Chen $X$, Gong J, Li D, Sun Z: Anticoagulant treatment is associated with decreased mortality in severe coronavirus disease 2019 patients with coagulopathy. J Thromb Haemost 2020; 18:1094-1099

98. Taubman-Ben-Ari O, Chasson $M$, Abu S, Weiss E: Distress and anxiety associated with COVID-19 among Jewish and Arab pregnant women in Israel. J. Reprod. Infant Psychol 2020; 38:340-348

99. The Lancet: The gendered dimensions of COVID-19. Lancet 2020; 395:1168

100. Toljan K: Letter to the editor regarding the viewpoint "Evidence of the COVID-19 virus targeting the CNS: tissue distribution, host-virus interaction, and proposed Neurotropic mechanism" ACS Chem Neurosci 2020; 11:1192-1194

101. Toraldo DM, DE Nuccio F, DE Benedetto M, Scoditti E: Obstructive sleep apnoea syndrome: a new paradigm by chronic nocturnal intermittent hypoxia and sleep disruption. Acta Otorhinolaryngol Ital 2015; 35:69-74

102. Troyer EA, Kohn JN, Hong S: Are we facing a crashing wave of neuropsychiatric sequelae of COVID-19? Neuropsychiatric symptoms and potential immunologic mechanisms. Brain Behav Immun 2020; 87:34-39

103. Uchino BN, Trettevik R, Kent de Grey RG, et al.: Social support, social integration, and inflammatory cytokines: a meta-analysis. Health Psychol 2018; 37:462-471

104. Varatharaj A, Thomas N, Ellul MA, et al.: Neurological and neuropsychiatric complications of COVID-19 in 153 
patients: a UK-wide surveillance study. Lancet Psychiatry 2020; 7:875-882

105. Virhammar J, Kumlien E, Fällmar D, et al.: Acute necrotizing encephalopathy with SARS-CoV-2 RNA confirmed in cerebrospinal fluid. Neurology 2020; 95:445-449

106. Walls AC, Park Y-J, Tortorici MA, Wall A, Mcguire AT, Veesler D: Structure, function, and antigenicity of the SARS-CoV-2 spike glycoprotein. Cell 2020; 181:281292.e6

107. Wang $C$, Pan $R$, Wan $X$, et al.: A longitudinal study on the mental health of general population during the COVID-19 epidemic in China. Brain Behav Immun 2020; $87: 40-48$

108. Wang $D, H u B, H u$, et al.: Clinical characteristics of 138 hospitalized patients with 2019 novel coronavirusinfected pneumonia in Wuhan, China. JAMA 2020; 323:1061-1069

109. Wang L, Shen Y, Li M, et al.: Clinical manifestations and evidence of neurological involvement in 2019 novel coronavirus SARS-CoV-2: a systematic review and metaanalysis. J Neurol 2020; 267:2777-89

110. Wang $Q, X u R$, Volkow ND: Increased risk of COVID-19 infection and mortality in people with mental disorders: analysis from electronic health records in the United States. World Psychiatry 2020

111. Wan $S, Y i Q$, Fan $S$, et al.: Characteristics of lymphocyte subsets and cytokines in peripheral blood of 123 hospitalized patients with 2019 novel coronavirus pneumonia (NCP). medRxiv 2020; 2020:2002.2010.20021832

112. Wasserman D, Iouse M, Wuestefeld A, et al.: Adaptation of evidence-based suicide prevention strategies during and after the COVID-19 pandemic. World Psychiatry 2020; 19:294-306

113. World Health Organization: Coronavirus Disease 2019 (COVID-19): Situation Report 2020. Available from: https://www.who.int/emergencies/diseases/novelcoronavirus-2019/advice-for-public

114. Xia H, Lazartigues E: Angiotensin-converting enzyme 2 in the brain: Properties and future directions. $J$ Neurochem 2008; 107:1482-1494

115. Xiao C: A Novel Approach of Consultation on 2019 Novel Coronavirus (COVID-19)-Related Psychological and Mental Problems: Structured Letter Therapy. Psychiatry Investig 2020; 17:175-176

116. Xie Q, Fan F, Fan XP, et al.: COVID-19 patients managed in psychiatric inpatient settings due to firstepisode mental disorders in Wuhan, China: clinical characteristics, treatments, outcomes, and our experiences. Transl Psychiatry 2020; 10:337

117. Yancy CW: COVID-19 and African Americans. JAMA 2020; 323:1891-2

118. Yao H, Chen JH, Xu YF: Patients with mental health disorders in the COVID-19 epidemic. Lancet Psychiatry 2020; 7:e21

119. Yockey LJ, Lucas C, Iwasaki A: Contributions of maternal and fetal antiviral immunity in congenital disease. Science 2020; 368:608-612

120. Zheng $W$ : Mental health and a novel coronavirus (2019nCoV) in China. J Affect Disord 2020; 269:201-202

121. Zhou F, Yu T, Du R, et al.: Clinical course and risk factors for mortality of adult inpatients with COVID-19 in Wuhan, China: a retrospective cohort study. Lancet 2020; 395:1054-62

Correspondence:

Yanfei He, MD

Health Management Center, the Sixth Medical Center, Chinese PLA General Hospital

NO.6 Fu Cheng Road, Hai Dian District, Beijing, 100048, China

E-mail: heyanfeilc@163.com 